\title{
Spontaneous remission of acute lymphoblastic leukemia: A case report
}

\author{
TIMM HÖRES, KNUT WENDELIN and KERSTIN SCHAEFER-ECKART \\ Department of Hematology and Medical Oncology, Paracelsus Medical University, D-90419 Nuremberg, Germany
}

Received May 3,2017; Accepted August 23, 2017

DOI: $10.3892 / 01.2017 .7288$

\begin{abstract}
Spontaneous remission (SR) in acute lymphoblastic leukemia (ALL) is a rare phenomenon, but the disease course and its underlying processes are of basic and clinical interest. Herein is reported the case of a pregnant, 31-year-old patient who developed ALL, followed by septic shock and SR of ALL. Information is summarized from earlier case reports and incidences of SR in ALL, to identify common patterns. Furthermore, the phenomenon of SR is compared with another disease variant of ALL, termed prodromal or preceding-ALL (pre-ALL). SR and the aleukemic phase in pre-ALL are associated with fever and/or sepsis and have similar kinetics and epidemiology. Therefore, pre-ALL not only closely resembles SR in ALL, but both conditions may represent a single disease entity. Production of pro-inflammatory cytokines and immune cell effects may induce temporary remission of ALL and the suppression of hematopoiesis. In contrast to SR in other types of cancer, all documented cases of SR in ALL were only transient. However, the disease can still be effectively treated with standard ALL therapies following relapse.
\end{abstract}

\section{Introduction}

Spontaneous remission (SR) in advanced-stage cancer is a rare but notable event, as it reveals the potential inherent reversibility of a fatal disease. Ignorance of physicians with regard to this phenomenon can provoke rejection of medical advice by the patient. It may even support the acceptance of quackery when cases of SR are attributed to dubious therapies (1). Although poorly understood, SR is a sufficiently documented phenomenon in several types of cancer, most frequently reported in malignant melanoma, neuroblastoma, nephroblastoma and malignant lymphoma (2). Everson (3)

Correspondence to: Dr Timm Höres, Department of Hematology and Medical Oncology, Paracelsus Medical University, Medizinische Klinik 5, Klinikum Nürnberg, Prof.-Ernst-Nathan-Straße 1, D-90419 Nuremberg, Germany

E-mail: timm.hoeres@klinikum-nuernberg.de

Key words: spontaneous remission, acute lymphoblastic leukemia, pancytopenia, sepsis, case report defined it as the "disappearance of a malignant tumor in the absence of all treatment or in the presence of therapy which is considered inadequate to exert a significant influence on neoplastic disease'. Positive effects of infectious disease on the course of leukemia were reported by Dock (4) as early as 1904. Bierman et al (5) described 11 cases of SR of ALL in detail, and identified recurring patterns. The frequency of SR in ALL may be as high as 4\% (6), but the overall incidence of ALL is low and such cases are now less frequently observed due to the rapid initiation of highly effective therapies. Herein is described a case of common-ALL followed by sepsis and a transient complete remission of leukemia. The observations are exemplary and indicate a close association of SR in ALL with an ALL disease-variant termed prodromal or preceding-ALL (pre-ALL).

\section{Case}

The patient, a pregnant (gravida I, 30 weeks of gestation) 31-year-old female, was admitted to the Department of Gynecology at Nuremberg Hospital (Nuremberg, Germany) in April 2014 with severe anemia, progressive fatigue and excessive sweating. Hemoglobin concentration $(\mathrm{Hb})$ was $5.0 \mathrm{~g} / \mathrm{dl}$ and the differential count indicated lymphocytosis and severe neutropenia. $\mathrm{Hb}$ was $13.2 \mathrm{~g} / \mathrm{dl}$ at the beginning of gestation and $9.7 \mathrm{~g} / \mathrm{dl}$ one month prior to admission. A total of four units of erythrocyte concentrates were transfused. Within four days the patient's leukocytes rose to $30.8 / \mathrm{nl}$, consisting of $91 \%$ mainly atypical lymphocytes consistent with leukemic blasts (Fig. 1). The patient then left hospital against medical advice.

On an ambulatory visit two days later, enlarged cervical lymph nodes were noted and the complete blood count (CBC) indicated progressive leukocytosis (Fig. 2). Flow cytometry analysis of peripheral blood samples demonstrated mainly CD45 low leukocytes that expressed CD10, CD19, HLA-DR, CD20, cytoplasmic CD79a and terminal deoxynucleotidyl transferase (Fig. 3). Chromosome analysis depicted a 46,XX karyotype in $7 / 17$, and a $46, \mathrm{XX}, \operatorname{del}(5)(\mathrm{q} 13 q 22)$ karyotype in 10/17 metaphases. Fluorescent in situ hybridization (FISH) analysis demonstrated an ACSL6 deletion in 85/100 interphase chromosomes. BCR-ABL1, MLL rearrangement or CDKN2A-deletions were not detected, and reverse transcription polymerase chain reaction was negative for BCR-ABL fusion products (Table I). Based on the flow cytometry findings, a diagnosis of common-ALL was determined. The 
patient refused bone marrow examination, as well as leukemia directed therapy or steroids. Over the next 8 days, the leucocyte count spontaneously dropped to $13 / \mathrm{nl}$; the thrombocyte count and $\mathrm{Hb}$ also decreased without treatment.

A total of 16 day following initial presentation, the patient was admitted with preterm uterine contractions, rupture of the membranes, fever, pancytopenia and elevated inflammation markers. The patient delivered a preterm, otherwise healthy, infant via cesarean section and antibiotics were administered for suspected neutropenic colitis. Simultaneously with clinical improvement, the patient's CBC and LDH began to normalize. Another 2 weeks later, no atypical lymphocytes were detectable on microscopic blood film examination, and flow cytometry demonstrated a decreased amount of CD45 low leukocytes. FISH was negative for ACSL-6 deletion in 100 analyzed interphase chromosomes (Table I). After 2 more weeks, flow cytometry of peripheral blood samples was negative for ALL, lymphadenopathy had resolved and the patient reported feeling well. At 6 weeks following discharge, the CBC was completely normal. However, after another 2 weeks, the patient presented with persistent rhinitis symptoms, swelling of the lips and urticarial rash. Prior to admission, the patient had been treated with penicillin and doxycycline for suspected sinusitis. LDH was elevated to $958 \mathrm{U} / 1$, the leukocyte count had increased to $31 / \mathrm{nl}$ with $50 \%$ atypical lymphocytes and hyperplasia of the adenoid tissue, associated with lymphoma, were noted.

A diagnosis of ALL relapse was determined, but the patient deferred all treatment except steroids, and emphasized their belief in recovery without chemotherapy. The patient received cefuroxime and a short course of prednisone. After 4 days, steroids had been tapered and the symptoms and lab results were again improving. However, flow cytometry analysis and molecular diagnostics (Table I) verified relapse. One month later, the patient presented with progressive symptoms from ALL, including anemia, thrombocytopenia, high blast count and abdominal pain. The following 6 weeks were characterized by clinical deterioration and infectious complications. During this time, treatment was limited to supportive measures, including antibiotics, transfusion of erythrocyte and thrombocytes concentrates and a short course of steroids, according to the patient's own explicit wishes. The patient then initiated treatment at a naturopathic clinic, from where they were urgently transferred because of life threatening sepsis. The patient received antibiotics and antifungal agents, and finally agreed to ALL-specific therapy, according to the German Multicenter Study Group on Adult Acute Lymphoblastic Leukemia (GMALL) study protocol 07/2003 (7). Disease manifestations rapidly improved, and induction phase I was completed with good adherence to the protocol standards.

Bone marrow biopsy verified complete molecular remission of ALL following induction therapy phase I. After three months, while undergoing induction therapy phase II and a modified consolidation therapy, the patient again developed life-threatening infectious complications with neutropenia, severe fatigue and marked nausea. Following consolidation phase II with one course of pegylated asparaginase, the patient declined further systemic therapy. A total of 14 months following the final ALL treatment, and 30 months after the initial diagnosis, bone marrow examination confirmed ongoing complete hematological and molecular remission. The full

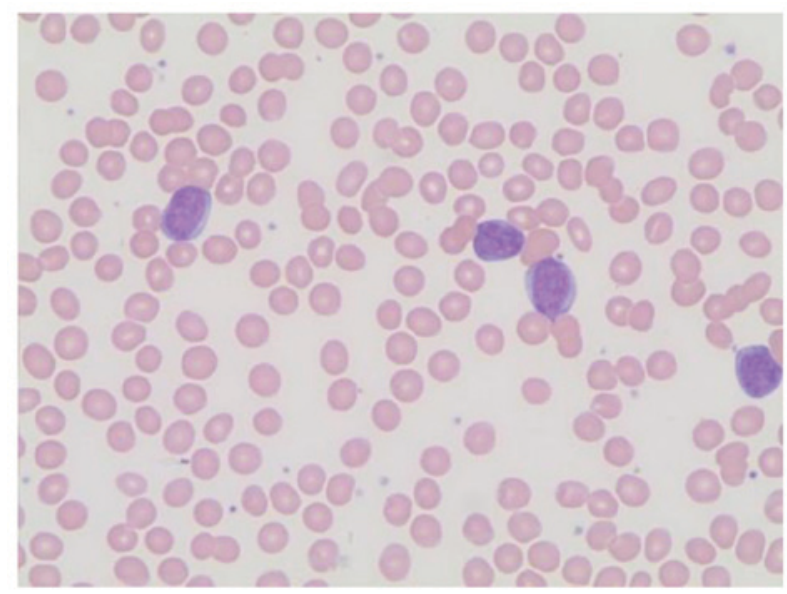

Figure 1. Initial diagnosis of ALL. Microscopic image of a Giemsa-Wright stained peripheral blood smear at presentation. Magnification, x100. ALL, acute lymphoblastic leukemia.

clinical course is summarized in Fig. 4. Chromosome analysis was supplemented by comparative-genomic-hybridization array analysis and FISH. Quantification is depicted as a rate of any certain characteristic from all evaluated metaphase or interphase chromosomes [X/Y]. FISH could not demonstrate a BCR-ABL1 or MLL rearrangement or a CDKN2A-deletion and reverse transcription polymerase chain reaction (PCR) was negative for BCR-ABL fusion products. Molecular screening-PCRs identified suitable molecular markers for the quantitative evaluation of minimal-residual-disease (MRD). The combination of tests for two different monoclonal rearranged genes: i) Immuno-globulin heavy chain (IGH DH2.21-JH3) and ii) $\gamma$-chain of the T-cell receptor (TRG $\mathrm{V} \gamma 2-\mathrm{J} \gamma \mathrm{P} 1)$ yields an assay sensitivity of $1 \times 10^{-5}(0,001 \%$ of nucleated cells).

\section{Discussion}

Case reports of SRs of ALL collected by Hirshberg and O'Regan (2) up to 1993, and the few published thereafter (8-12), typically describe a pattern of feverish or septic disease followed by quick but transient remission. If steroids are used, remissions are usually not considered spontaneous due to their well-characterized anti-leukemic effect (13). The use of antibiotics and blood transfusions is frequently described, but no individual supportive treatment has been identified as causally associated with the development of SR. It is noteworthy that pre-ALL, a disease variant of ALL that occurs in $2 \%$ of all ALL cases (14-16), resembles a number of features of SR: The majority of patients are female, and present with fever, fatigue and hypoplastic bone marrow or cytopenia. Occasionally, lymphadenopathy, hepatosplenomegaly and relative lymphocytosis are observed. Although mostly diagnosed with aplastic anemia, patients can recover and develop complete, if transient, recovery of normal bone marrow function. In the majority of cases, 1-14 months following remission patients present with early B-lymphoblastic leukemia (17-19). The present case observations support an earlier report by Boonchalermvichian et al (10), and suggest that pre-ALL and SR in ALL may actually represent different stages of the same 


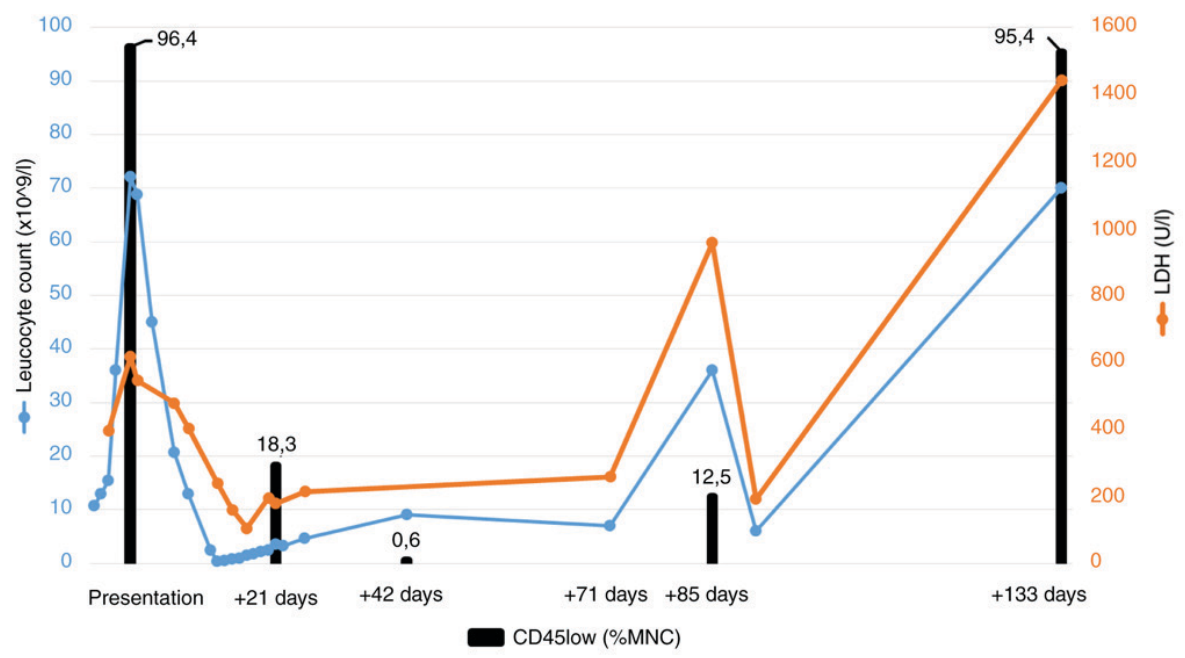

Figure 2. Course of disease. Changes in leucocyte count (10\%l), LDH (U/1) and rate of CD45 low (\%MNC) leucocytes in the peripheral blood by flow cytometry. Timeline is presented as the days following presentation. $\mathrm{LDH}$, lactate dehydrogenase; $\mathrm{CD}$, cluster of differentiation; MNC, mononuclear cell.
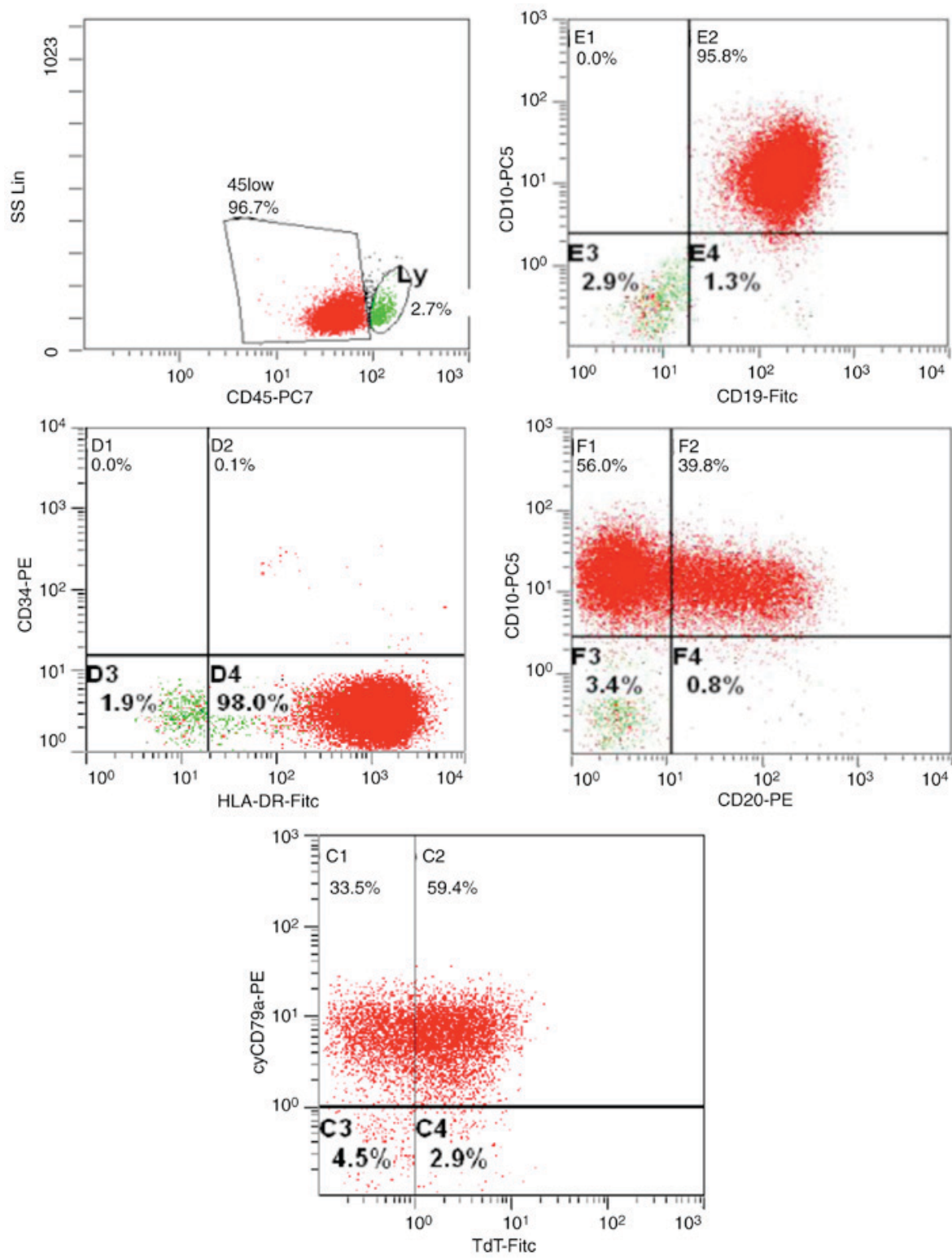

Figure 3. Flow cytometry analysis of peripheral blood at the time of initial ALL diagnosis. ALL, acute lymphoblastic leukemia; CD, cluster of differentiation; PE, phycoerythrin; FITC, fluorescein isothiocyanate; TdT, terminal deoxynucleotidyl transferase. 
Table I. Cytogenetics and molecular tests performed on peripheral blood and bone marrow samples.

\begin{tabular}{lllcl}
$\begin{array}{l}\text { Days post } \\
\text { presentation }\end{array}$ & \multicolumn{1}{c}{ Material } & \multicolumn{1}{c}{ Cytogenetic/FISH } & MRD-level & \multicolumn{1}{c}{ Comment } \\
\hline$+8 \mathrm{~d}$ & Peripheral blood & $46, \mathrm{XX}[7 / 17] ;$ 46,XX,del(5)(q13q22) [10/17] & $3 \times 10^{-1}$ & \multicolumn{1}{c}{ Initial diagnosis } \\
$+8 \mathrm{~d}$ & Peripheral blood & FISH: ACSL6-deletion [85/100] & $3 \times 10^{-1}$ & \multicolumn{1}{l}{ Initial diagnosis } \\
$+26 \mathrm{~d}$ & Peripheral blood & $46, \mathrm{XX}[6 / 6] /$ FISH: ACSL6-deletion [0/100] & Not determined & Spontaneous remission \\
$+91 \mathrm{~d}$ & Peripheral blood & $46, \mathrm{XX}[8 / 10] ;$ 46,XX,del(5)(q21q31) [2/10] & $2 \times 10^{-2}$ & Relapse \\
$+133 \mathrm{~d}$ & Peripheral blood & $46, \mathrm{XX}[11 / 11] /$ FISH: ACSL6-Deletion [0/100] & Not determined & Progression \\
$+194 \mathrm{~d}$ & Peripheral blood & Not determined & $5 \times 10^{-4}$ & Following pre-phase \\
$+194 \mathrm{~d}$ & Bone marrow & Not determined & $1 \times 10^{-2}$ & Following pre-phase \\
$+224 \mathrm{~d}$ & Bone marrow & FISH: ACSL6-deletion [0/100] & $<5 \times 10^{-5}$ & Following induction I \\
$+452 \mathrm{~d}$ & Bone marrow & Not determined & $<5 \times 10^{-5}$ & Start consolidation \\
$+553 \mathrm{~d}$ & Bone marrow & Not determined & $<5 \times 10^{-5}$ & Follow-up \\
$+924 \mathrm{~d}$ & Bone marrow & Not determined & $<5 \times 10^{-5}$ & Follow-up
\end{tabular}

FISH, fluorescence in situ hybridization; MRD, minimal residual disease.

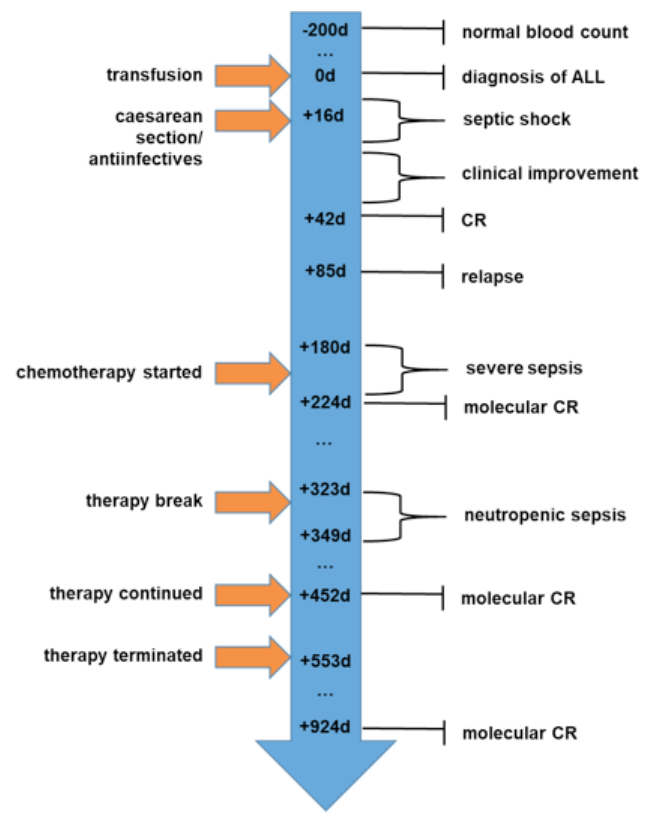

Figure 4. Timeline of clinical events and therapeutic interventions, listed as the days following presentation. CR, complete remission.

ALL disease variant. Distinctive classification of pre-ALL and SR in ALL can be attributed to variations in the time of presentation and the limited sensitivity of current diagnostic tests (Fig. 5).

An important limitation of this report is the lack of bone marrow biopsy data prior to the initiation of leukemia-specific treatment and minimal residual disease studies following SR. More sensitive tests would have been helpful to better characterize the disease and the degree of SR. The concept that in cases of pre-ALL an overt malignant condition may exist initially undetected but potentially traceable by molecular tests, is supported by numerous retrospective analyses $(16,19-21)$, and by a case report of a patient who received allogenic stem cell transplantation for suspected severe aplastic anemia and later developed ALL originating from hosts cells (22). A

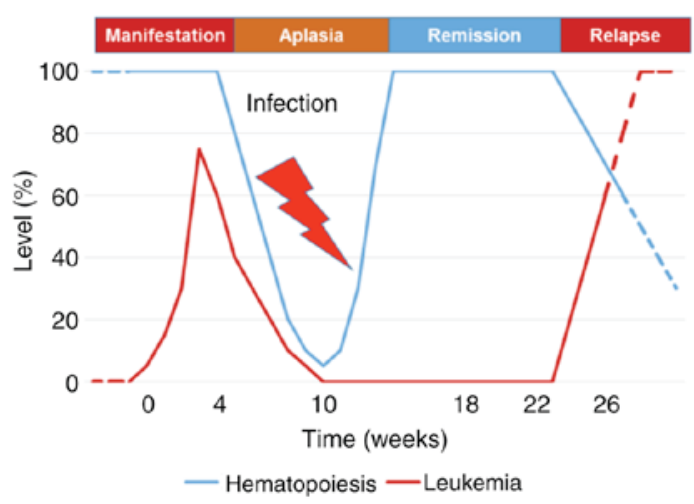

Figure 5. Model of spontaneous remission in ALL and preceding-ALL kinetics. Small variations in the time of observation or the diagnostic sensitivity could account for the distinctive classification of the same entity as either preceding-ALL or as spontaneous remission of ALL. ALL, acute lymphoblastic leukemia.

review of the literature did not reveal any specific patterns of cytogenetic abnormalities in SR or pre-ALL.

The 6 case reports of SR of ALL post-1993 contained 5 chromosome analyses, of which 2 were normal (8-12). In 64 reported cases of childhood or adult pre-ALL $(10,14-20,23-38)$ only 10 chromosome analyses were reported in the aleukemic phase, of which 9 were normal. In the following overt ALL phase, 14 cytogenetic tests were performed, of which 8 were normal. The described cytogenetic abnormalities included complex karyotypes, numeric alterations as well as translocation and derivations in SR and in pre-ALL. A recently published study did not report classical cytogenetic studies but retrospectively analyzed specimens of the aleukemic phase of 8 cases of pre-ALL, and detected ETV6-RUNX1 fusions in bone marrow cells that were also present during overt ALL (21). The study suggested that in pre-ALL established (pre-)leukemic clones are temporarily controlled by the immune system until eventually dominant subclones are selected and induce clinical ALL (21). 
Various mechanisms, including apoptosis, endogenous steroid effects and immunological reactions following infections, have been proposed to explain occurrence of SR (39). The assumption that anti-leukemic immune reactions serve a major role in many cases may be primarily derived from the observation that infections and fever often precede remission. Additionally, an accumulation of T-cells was reported in histological specimens from the bone marrow of patients with pre-ALL or SR of ALL $(10,17)$. Cytokines, such as tumor necrosis factor- $\alpha$ or interferon- $\gamma$, which are produced during infection, are potent modulators of immune cells and can induce direct and indirect antitumor activity (40). Pro-inflammatory cytokines are also capable of suppressing normal hematopoiesis under certain conditions (41), providing a possible linkage for aplasia and remission. It is suggested that pro-inflammatory cytokines and immune cell activity during severe infection shift the balance of pro-leukemia towards an anti-leukemia environment, and induce temporary remission of ALL and a suppression of hematopoiesis.

Further elucidation of the processes underlying SR is required, as it may translate into novel treatment strategies for ALL. Studies that investigate the mechanisms of SR are challenging to perform, but one feasible approach is the implementation of register studies and the bio-banking of material to facilitate retrospective analyses. A relevant clinical question is how to handle cases of SR in ALL. An early implementation of cytotoxic therapy during SR in ALL may be favorable, as the remissions are transient. However, there is no direct evidence to support this approach, and the few recent case reports have indicated that the majority of patients with SR of ALL are cured following relapse using established therapies. Thus, the prognosis of patients with pre-ALL appears to be identical to that of classical ALL cases $(15,38,42)$.

\section{References}

1. Lowy AD Jr and Erickson ER: Spontaneous 19-year regression of oat cell carcinoma with scalene node metastasis. Cancer 58 : 978-980, 1986

2. Hirshberg C and O'Regan B: Spontaneous remission: An annotated bibliography. Inst Noetic Sci: 713, 1993.

3. Everson TC: Spontaneous regression of cancer. Ann N Y Acad Sci 114: 721-735, 1964.

4. Dock G: The influence of complicating diseases upon leukemia. Am J Med Sci 127: 563-592, 1904.

5. Bierman HR, Crile DM, Dod KS, Kelly KH, Petrakis NL, White LP and Shimkin MB: Remissions in leukemia of childhood following acute infectious disease: Staphylococcus and streptococcus, varicella, and feline panleukopenia. Cancer 6 : 591-605, 1953 .

6. Diamond LK and Luhby AL: Pattern of 'spontaneous' remissions in leukemia of childhood observed in 26 of 300 cases. Am J Med 10: 238-239, 1951.

7. Raff T, Gökbuget N, Lüschen S, Reutzel R, Ritgen M, Irmer S, Böttcher S, Horst HA, Kneba M, Hoelzer D, et al: Molecular relapse in adult standard-risk ALL patients detected by prospective MRD monitoring during and after maintenance treatment: Data from the GMALL 06/99 and 07/03 trials. Blood 109: 910-915, 2007.

8. Purohit A, Aggarwal M, Kumar S, Seth T, Mishra P, Mahapatra M, Saxena R, Sharma R, Singh PK and Venkateshan S: Spontaneous remission of adult acute lymphoblastic leukemia: A very rare event. Indian J Hematol Blood Transfus 31: 159-160, 2015.

9. Pluchart C, Munzer M, Mauran P and Abély M: Transient remission of childhood acute lymphoblastic and myeloid leukemia without any cytostatic treatment: 2 case reports and a review of literature. J Pediatr Hematol Oncol 37: 68-71, 2015.
10. Boonchalermvichian C, Xie Y, Brynes RK and Siddiqi IN: Spontaneous, transient regression of B lymphoblastic leukemia in an adult patient: A variant presentation of prodromal/pre-ALL. Leuk Res 36: e57-e59, 2012.

11. Chen RL and Chuang SS: Transient spontaneous remission after tumor lysis syndrome triggered by a severe pulmonary infection in an adolescent boy with acute lymphoblastic leukemia. J Pediatr Hematol Oncol 31: 76-79, 2009.

12. Yoruk A, Erguven M, Celiker E, Aki H, Timur C, Yuksel E and Ozkan H: Spontaneous remission of acute lymphoblastic leukemia with mediastinal mass. Pediatr Hematol Oncol 25: 181-186, 2008.

13. Inaba $\mathrm{H}$ and Pui $\mathrm{CH}$ : Glucocorticoid use in acute lymphoblastic leukaemia. Lancet Oncol 11: 1096-1106, 2010.

14. Breatnach F, Chessells JM and Greaves MF: The aplastic presentation of childhood leukaemia: A feature of common-ALL. Br J Haematol 49: 387-393, 1981.

15. Armata J, Grześkowiak-Melanowska J, Balwierz W, Najbar-Pabian A and Pawlik-Niesytto E: Prognosis in acute lymphoblastic leukemia (ALL) in children preceded by an aplastic phase. Leuk Lymphoma 13: 517-518, 1994.

16. Horsley SW, Colman S, McKinley M, Bateman CM, Jenney M, Chaplin T, Young BD, Greaves M and Kearney L: Genetic lesions in a preleukemic aplasia phase in a child with acute lymphoblastic leukemia. Genes Chromosomes Cancer 47: 333-340, 2008.

17. Li Q, Chen Z, You Y and Zou P: Transient pancytopenia preceding acute lymphoblastic leukemia with positive Philadelphia chromosome. Leuk Res 32: 1317-1320, 2008.

18. Villarreal-Martínez L, Jaime-Pérez JC, Rodríguez-Martínez M, González-Llano O and Gómez-Almaguer D: Acute lymphoblastic leukemia of childhood presenting as aplastic anemia: Report of two cases. Rev Bras Hematol Hemoter 34: 165-167, 2012.

19. Lynggaard LS, Marquart HV, Kjeldsen E, Madsen HO and Hasle H: Acute lymphoblastic leukemia presenting with pancytopenia followed by a 14-month-long period of transient remission possibly supporting the adrenal hypothesis of leukemogenesis. J Pediatr Hematol Oncol 38: e271-e273, 2016.

20. Robak T, Bartkowiak J, Urbańska-Rys H, Szmigielska-Kaplon A, Strzelecka B and Chojnowski K: Acute lymphoblastic leukemia in adult first manifested as severe aplastic anemia-role of molecular analysis in correct diagnosis. Leuk Lymphoma 43: 1147-1152, 2002.

21. Zimmermannova O, Zaliova M, Moorman AV, Al-Shehhi H, Fronkova E, Zemanova Z, Kalina T, Vora A, Stary J, Trka J, et al: Acute lymphoblastic leukemia with aleukemic prodrome: Preleukemic dynamics and possible mechanisms of immunosurveillance. Haematologica 102: e225-e228, 2017.

22. Klingemann HG, Storb R, Sanders J, Deeg HJ, Appelbaum FR and Thomas ED: Acute lymphoblastic leukaemia after bone marrow transplantation for aplastic anaemia. Br J Haematol 63: 47-50, 1986.

23. Bernard $\mathbf{J}$ and Schaison G: Transitory bone marrow failure: A series of 13 preleukemic cases in children. J Pediatr Hematol/Oncol 2, 1980.

24. de Vaan GA and van Oostrom CG: The aplastic presentation of childhood leukaemia. Br J Haematol 50: 712-713, 1982.

25. Dharmasena F, Littlewood T, Gordon-Smith EC, Catovsky D and Galton DA: Adult acute lymphoblastic leukaemia presenting with bone marrow aplasia. Clin Lab Haematol 8: 361-364, 1986.

26. Liang R, Chan TK and Todd D: Childhood acute lymphoblastic leukaemia and aplastic anaemia. Leuk Lymphoma 13: 411-415, 1994.

27. Liang R, Cheng G, Wat MS, Ha SY and Chan LC: Childhood acute lymphoblastic leukaemia presenting with relapsing hypoplastic anaemia: Progression of the same abnormal clone. Br J Haematol 83: 340-342, 1993.

28. Liang Y, Ding L, Li X, Wang W and Zhang X: Transient pancytopenia preceding adult acute lymphoblastic leukemia with chromosomal abnormalities including the Philadelphia chromosome: A case report and review of the literature. Oncol Lett 10: 3789-3792, 2015

29. Matloub YH, Brunning RD, Arthur DC and Ramsay NK: Severe aplastic anemia preceding acute lymphoblastic leukemia. Cancer 71: 264-268, 1993

30. Morley AA, Brisco MJ, Rice M, Snell L, Peng LM, Hughes E, Neoh SH and Sykes PJ: Leukaemia presenting as marrow hypoplasia: Molecular detection of the leukaemic clone at the time of initial presentation. Br J Haematol 98: 940-944, 1997.

31. Nakamori Y, Takahashi M, Moriyama Y, Httori A, Shibata A, Watanabe T and Oda Y: The aplastic presentation of adult acute lymphoblastic leukemia. Br J Haematol 62: 782-783, 1986. 
32. Reid MM and Summerfield GP: Distinction between aleukaemic prodrome of childhood acute lymphoblastic leukaemia and aplastic anaemia. J Clin Pathol 45: 697-700, 1992.

33. Saarinen UM and Wegelius R: Preleukemic syndrome in children. Report of four cases and review of literature. Am J Pediatr Hematol Oncol 6: 137-145, 1984.

34. Sarangi JN, Kashyap R, Choudhry VP, Mishra DK, Saxena R, Gurbaxani S and Bhargava M: Severe aplastic anemia evolving into T cell acute lymphoblastic leukemia. Eur J Haematol 63 269-271, 1999.

35. Sohn SK, Suh JS, Lee J and Lee KB: Pancytopenic prodrome (pre-ALL) of acute lymphoblastic leukemia in adults: Possible pathogenesis. Korean J Intern Med 13: 64-67, 1998.

36. Takeuchi M, Soda R, Takahashi K, Kimura F, Lai M and Ueda Y: Philadelphia chromosome positive acute lymphocytic leukemia arising from aplastic anemia. Am J Hematol 63: 161-162, 2000.

37. Toledano SR: Preleukemic phase in childhood acute lymphoblastic leukemia. J Pediatr 91: 507-508, 1977.

38. Wegelius R: Bone marrow dysfunctions preceding acute leukemia in children: A clinical study. Leuk Res 16: 71-76, 1992.
39. Papac RJ: Spontaneous regression of cancer. Cancer Treat Rev 22: 395-423, 1996.

40. Puré E, Allison JP and Schreiber RD: Breaking down the barriers to cancer immunotherapy. Nature immunology 6: 1207-1210, 2005.

41. Selleri C, Sato T, Anderson S, Young NS, Maciejewski JP: Interferon-gamma and tumor necrosis factor- $\alpha$ suppress both early and late stages of hematopoiesis and induce programmed cell death. J Cell Physiol 165: 538-546, 1995.

42. Hasle H, Heim S, Schroeder H, Schmiegelow K, Ostergaard E and Kerndrup G: Transient pancytopenia preceding acute lymphoblastic leukemia (pre-ALL). Leukemia 9: 605-608, 1995.

his work is licensed under a Creative Commons

Attribution-NonCommercial-NoDerivatives 4.0 International (CC BY-NC-ND 4.0) License. 